\title{
Fuzzy Color Image Segmentation using Watershed Transform
}

\author{
Noël Richard and Christine Fernandez-Maloigne \\ XLIM Laboratory, CNRS JUR 7252, University of Poitiers France \\ Email: $\{$ noel.richard, christine.fernandez $\} @$ univ-poitiers.fr \\ Cristian Bonanomi and Alessandro Rizzi \\ Dipartimento di Informatica, Università degli Studi di Milano, Italy \\ Email: \{cristian.bonanomi, alessandro.rizzi\}@unimi.it
}

\begin{abstract}
In this paper, we present a segmentation technique which joins a multi-scale texture based approach and a fuzzy segmentation method using the color gradient of Di Zenzo. Thus maps of homogeneous texture patterns are enhanced by edge localization. A topographic distance is used to calculate membership degrees to each region. Both lead to segmentation using a step of watershed flooding, with few parameters to set. Moreover computation complexity has been reduced to allow treatments of images in full size and colors.
\end{abstract}

Index Terms - color image segmentation, fuzzy image segmentation, color watershed, color gradient, multi-scale texture segmentation

\section{INTRODUCTION}

A recurrent problem in image segmentation is variability and complexity, especially in natural or art scenes, which make the segmentation process very difficult to optimize [1]-[3]. However, this could be bypassed with the cooperation of different methods.

We present in this paper some new features in order to develop a multi-scale approach keeping contours and texture information for fuzzy segmentation of color images [4]. This could be very useful in order to treat art painting from medieval buildings. This kind of images has a specific noise due to time alteration. Moreover, we aim at finding objects in images with 10 millions pixels and thousands of color. In this sense, we combine a wellknown texture based technique (JSEG) with fuzzy information obtained with Di Zenzo's color gradient norm [5].

\section{WATERSHED SEGMENTATION PRINCIPLE}

In the field of mathematical morphology, watershed transforms are often used in order to detect objects of interest in images [6]. The watershed transformation was originally proposed in late of 70's as a tool for segmenting gray-scale images [7]. Nowadays it is used as an elemental step in many powerful color segmentation procedures [8]. The watershed constitutes one of the main

Manuscript received June 4, 2013; revised August 7, 2013. concepts of Mathematical Morphology. The watershed transform can be classified as a region-based segmentation approach. The intuitive idea underlying this method comes from geography. Since any gray-scale image can be considered as topographic surface: we regard the intensity of a pixel as altitude of the point. Let us imagine the surface of this relief being immersed in still water, with holes created in local minima. Water fills up the dark areas "the basins" starting at these local minima. Where waters coming from different basins meet we will build dams. When the water level has reached the highest peak in the landscape, the process is stopped. As a result, the landscape is partitioned into regions or basins separated by dams, called watershed lines or simply watersheds, as we can see on Fig. 1. Many different watershed algorithms have been proposed until today [9].

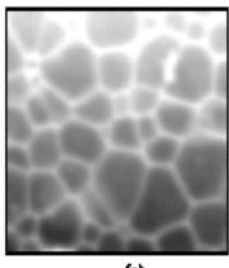

(a)

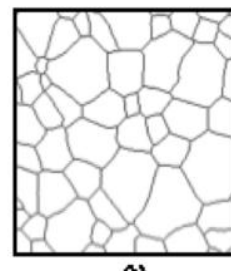

(b)

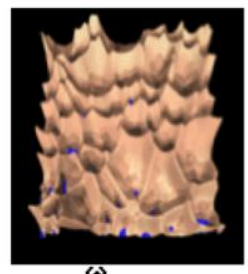

(c)
Figure 1. Image (a) shows the initial image, image (b) shows the result, image (c) shows a 3D representation.

This process produces an important over-segmentation, as we can see on Fig. 2, usually resolved by the use of a markers image. However, this process involves another costly step in image treatments.

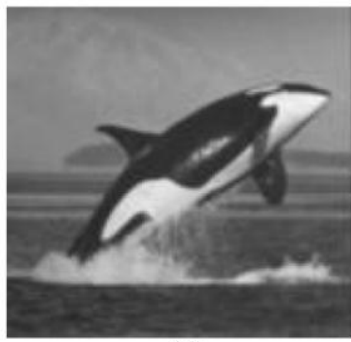

(a)

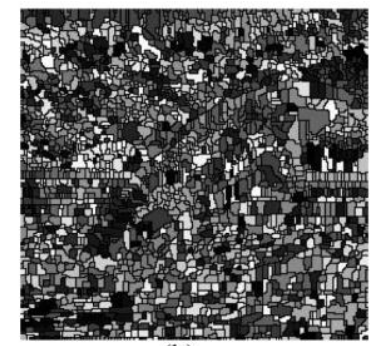

(b)
Figure 2. Image (a) shows the initial image, image (b) shows the result Image. Different basins are indicated by distinct grey values. 


\section{OUR Methodology}

A multi-scale approach combined with region merging during the watershed flooding transform permits to avoid the step of markers definition. First of all we compute an energy image by calculating the mean of each color class in the image. This criterion called $\mathrm{J}$, is introduced by Deng [10]. It detects homogeneous regions in an image, using a texture discontinuity based approach. This measure can be calculated for each pixel in the entire image. In the original Deng's version, the color information is only used in a pre-processing quantization step; all colors are considered as classes. Thus, the segmentation process is dependent on the quantization but is not connected to it. The $\mathrm{J}$ computation uses the fact that a color texture is uniform on the area of study if the gravity centers of the colors are confounded and if their dispersions are the same. Therefore, the $\mathrm{J}$ values are computed using the center positions of classes and their spatial layouts. More precisely, if there are $\mathrm{n}$ colors $\{\mathrm{C} 1, \ldots, \mathrm{Cn}\}$ in the studied area $\mathrm{A}$. Let $\mathrm{G}$ be the mean location of the area and $\mathrm{Gi}$ the color $\mathrm{i}$. Let $\mathrm{D}_{\mathrm{t}}$ be the dispersion of the global area: $D_{t}=\Sigma_{p} \varepsilon A d(p, G)$ where $d$ is the used distance $\left(\mathrm{L} 1, \mathrm{~L} 2\right.$. . . ). Let $\mathrm{D}_{\text {ri }}$ be the relative dispersion of the color $\mathrm{i}$ : $\mathrm{D}_{\mathrm{ri}}=\Sigma_{\mathrm{p} \varepsilon \mathrm{Ci}} \mathrm{d}(\mathrm{p}, \mathrm{Gi})$. Then:

$$
J=\frac{D_{t}-\sum_{i=1}^{n} D_{r i}}{D_{t}}
$$

Fig. 3 shows some examples of $\mathbf{J}$ values on a small neighborhood. It demonstrates the increase of $\mathrm{J}$ while the texture break becomes more important. From left to right, the centers of the colors are more and more distant from the mean location of the neighborhood; therefore $\mathrm{J}$ becomes higher.

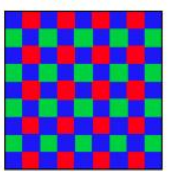

$\mathrm{Dt}=\mathrm{Dr}$ $\mathrm{J}=\mathbf{0}$

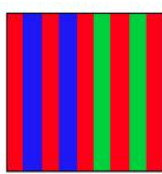

Dt $>$ Dr $\mathrm{J}=0.088$

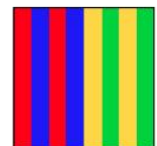

$\mathrm{Dt} \gg \mathrm{Dr}$ $\mathrm{J}=0.23$

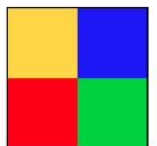

$\mathrm{Dt} \gg \mathrm{Dr}$ $\mathrm{J}=0.51$
Figure 3. Examples of J values with an increasing texture break

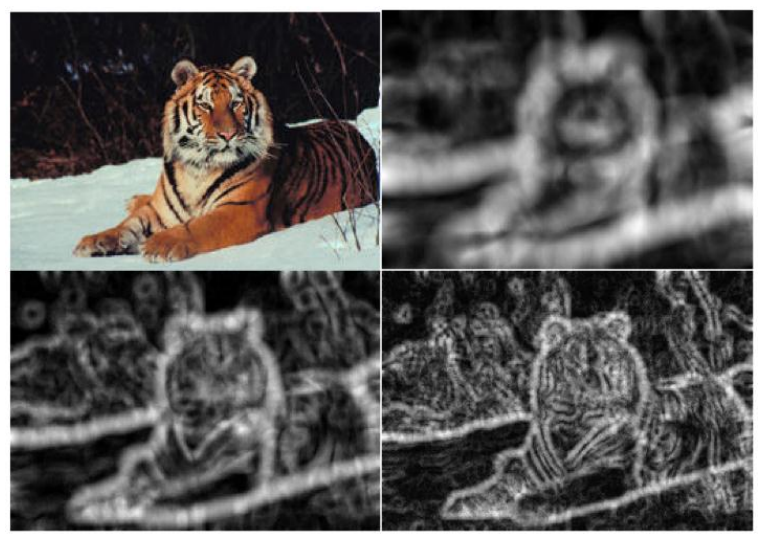

Figure 4. Examples of $\mathrm{J}$ maps (are used to obtain the segmentations of the tiger on the Fig. 6).
We have modified this criterion to keep all the color information in Dombre and al. [11]. Consequently a Jimage is provided. Computing the criterion at different scales we obtain a grey scale image representing the more important texture breaks, or the finest ones (cf. Fig. 4). This property is used to build a multi-scale algorithm. The generation of the regions is based on a simple watershed technic applied on the various J maps. We start with a large window and we decrease its size to refine the segmentations. The previous segmentation levels are used to increase the precision of the watershed process. Hence, this approach allows us to obtain coherent segmentations and a multi-scale representation.

Fig. 5 shows some segmentation examples. A merging criterion based on color and/or texture is used in order to decrease the number of similar adjacent regions.
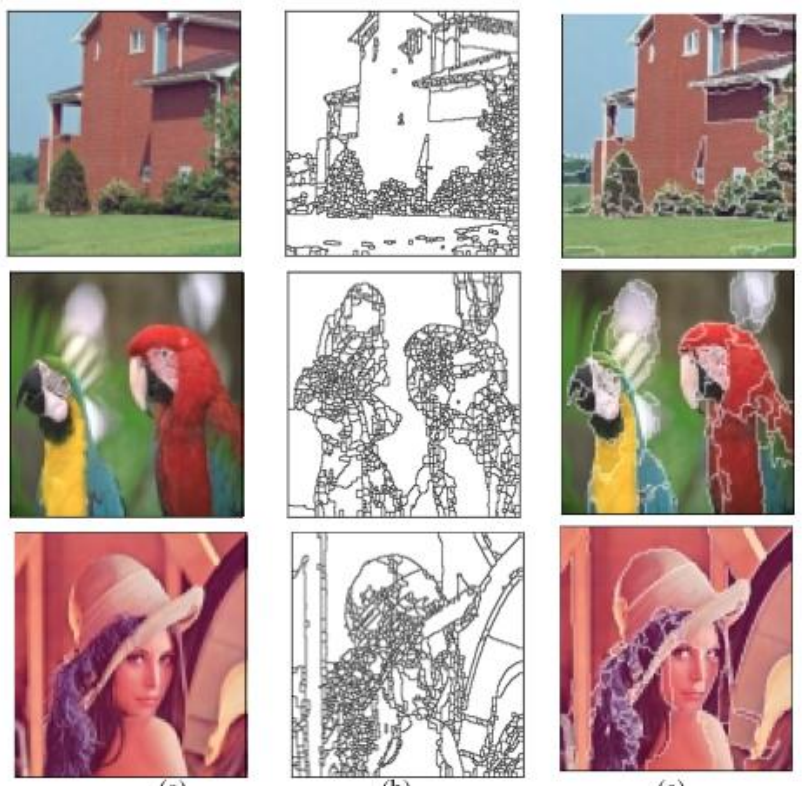

(c)

Figure 5. Image (a) shows the original, image (b) shows the oversegmentation with a traditional watershed algorithm, image (c) show Dombre's algorithm applied on image (a)

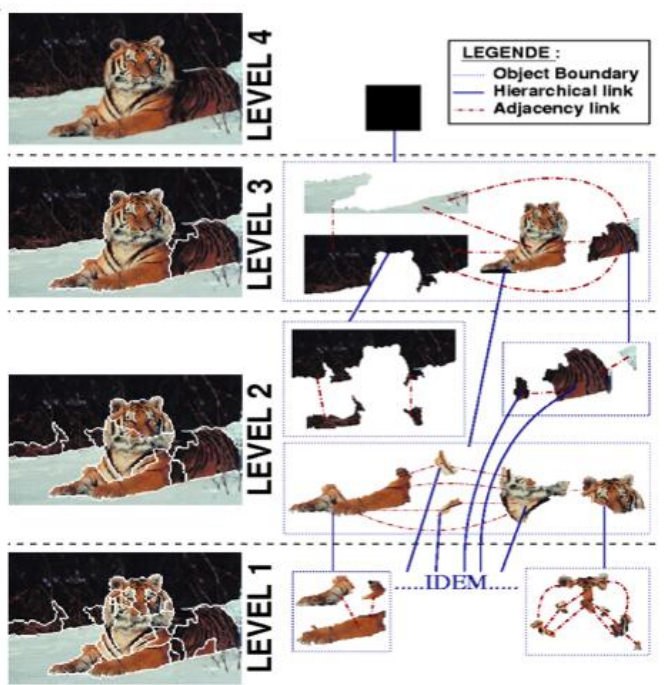

Figure 6. Pyramidal graph example based on a real segmentation 
Moreover, we have solved the problem of spatial arrangement and objects inclusion with a pyramidal graph structure [12], as we can see in Fig. 6. This new representation system, based on a graph, mixes the region and border based approaches. Regions provide a description of the different objects (or part of objects) while borders describe the interactions between the objects.

The weakness of such a processing is the lack of accuracy close to boundaries. So we propose to complete the $J$ calculation by the use of Di Zenzo's color gradient norm [5]. Here are the three ways we propose to achieve this task: thresholding, meaning, and weighting. The first one can be applied keeping either gradient information over a selected threshold, either low J-values. We can get homogeneous regions as well as good edge localization for line detection. With the other combinations noise involved by gradient can be considerably reduced. The third method is realized with multiplication of grey scale gradient norm image by normalised J-map. Then this optimized J-image (Fig. 7) is used for the region-growing step. As a result we obtain a map that is more efficient for the watershed part of our algorithm, keeping good precision of gradient close to boundaries as well as texture discontinuities over the regions.
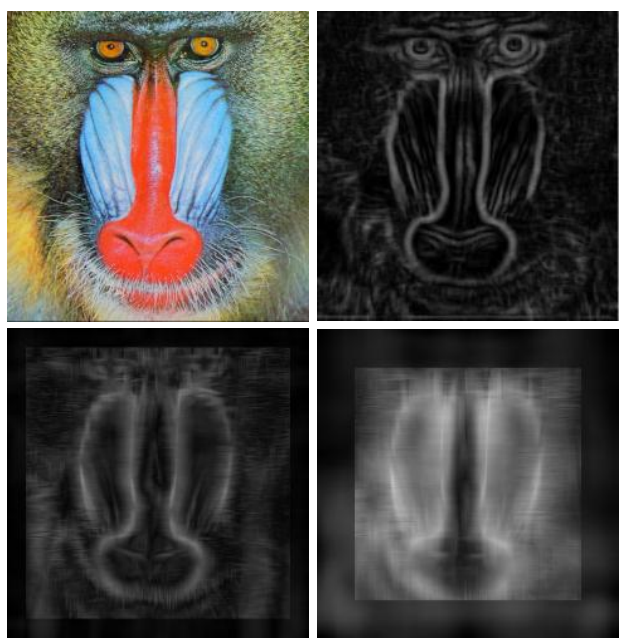

Figure 7. Original 512×512 image, then weighed J-image at scale 9, 64, and 128.

\section{RESULTS}

Finally, in the proposed method, the watershed transform is applied using every local minima of the weighted map as a seed from where we can start the iterative flooding of the map. Our watershed is based on Vincent algorithm [13]. During this step, some regions could be merged like in Andrade method to avoid the use of markers [14]. When two lakes meet, if one of them has a depth or a size lower than fixed thresholds, merging is proceeded. Nevertheless, we suggest taking into account the distance between depths rather than using a simple threshold, which is furthermore very difficult to set for a large variety of images. Moreover, fuzzy information is computed during the weighted J-image immersion, using Vieira formulation of membership degrees [15]. Minima are the core of the fuzzy region and have therefore maximal value of membership to this region. Then degrees are provided by (2), minimizing the cost $\mathrm{C}$ on a path. This computation is based on a criterion that includes spatial nearness and our weighted J-image magnitude. Spatial measure is a topographic distance representing the shortest $3 \mathrm{D}$ path:

$$
\mathrm{C}\left(\mathrm{p}_{\mathrm{i}-1}, \mathrm{p}_{\mathrm{i}}\right)=\mathrm{k} .\left|\mathrm{J}\left(\mathrm{p}_{\mathrm{i}}-1\right)-\mathrm{J}\left(\mathrm{p}_{\mathrm{i}}\right)\right|+\mathrm{D}\left(\mathrm{p}_{\mathrm{i}-1}, \mathrm{p}_{\mathrm{i}}\right)
$$

where $J$ is our weighted map, $p_{i}$ a pixel of the path, $D$ an Euclidean distance.

Our multi-scale approach permits to obtain different levels of precision. Depending on the map used, the process provides different segmentations. Thus, we construct a hierarchical pyramidal graph. This gives knowledge about composition of segmented objects at high scales, as we can see on Fig. 8:

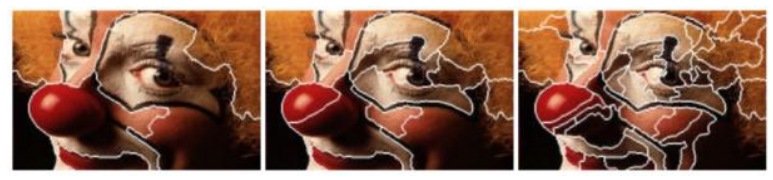

Figure 8. Final different levels of segmentation.

\section{CONCLUSION}

This paper features a new method for extending the JSEG algorithm, reducing the computation cost and aiming at solving the issue of natural noise in texture patterns. The segmentation process is based on a multiscale region growing from maps that takes into account both $\mathbf{J}$ criteria and color gradient information. A small number of parameters is needed by our algorithm, and are less sensitive to small variations than in the original approaches.

A recursive reformulation of the J-image algorithm allows to treat large size images. With this improvement, there is no need of a perceptual quantification. In the original approaches, we had to reduce the color number about to ten, losing colorimetric information. Moreover, a sub-sampling/smoothing process is used whenever the scale is improved. With our method we can work with the entire colour panel, and keep color information at each scale and for all the pixels. So images with millions of pixels and colours can be segmented in a few seconds.

Texture pattern discontinuity is improved by contour detection, and this combination decreases the number of scales to compute. Moreover holes in boundaries can be eliminated and we attenuate noise induced by the gradient norm, particularly when a pixel is far away from boundaries. We reduce over-segmentation and watershed computation time because of higher contrast in our energy cards.

Computation time for a $512 \times 512$ images coded in RGB is about 3 s for a mask $17 \times 17$ on a Pentium-M 1,8 Ghz and about $45 \mathrm{~s}$ at scale 64 .

Future work will try to increase multi-scale properties between different scales to enhance accuracy and to define automatically the parameters needed. We want also to include perceptual color distance in the 
computation of the $\mathrm{J}$ criterion and to use fuzzy information for image indexing and pattern recognition in complex image databases that can be useful in many applications.

\section{ACKNOWLEDGMENT}

This work was supported in part by a grant from Region Poitou-Charentes, France

\section{REFERENCES}

[1] C. Fernandez-Maloigne (Ed.). Advanced Color Image Processing and Analysis. Springer, July, 2012

[2] C. Fernandez-Maloigne, F. Robert-Inacio, and L. Macaire (Ed.), Numerical Color Imaging, ISTE Ltd/John Wiley \& Sons, 2012.

[3] N. Richard, A. Ledoux, A. Capelle-Laizé, and C. FernandezMaloigne, "Toward a valid image processing system through color standards," presented at CIE Midterm conference, Paris, France, April 14-16, 2013

[4] S. Makrogiannis, I. Vanhamel, S. Fotopoulos, H. Sahli, and J. P. H. Cornelis, "Watershed-based multiscale segmentation method for color images using automated scale selection," J. Electron. Imaging, vol. 14, no. 3, July, 2005.

[5] S. Di Zenzo, "Note: A note on the gradient of a multi-image," Computer Vision, Graphics, and Image Processing, vol. 33, no. 1, pp. 116-125, 1986.

[6] J. B. T. M. Roerdink and A. Meijster, "The watershed transform: Definitions, algorithms and parallelization strategies," Fundamenta Informaticae, vol. 41, pp. 187-228, 2000.

[7] S. Beucher and C. Lantuéjoul, "Use of watersheds in contour detection," presented at International Workshop on Image Processing, Real-Time Edge and Motion Detection/Estimation, France, September, 1979.

[8] J. Chanussot and P. Lambert. Watershed approach for color image segmentation. Available: http://gipsawww.ampere.inpg.fr/ jocelyn.chanussot/publis/ieee_nsip_99_cha nuss_watershed.pdf

[9] A. R. Chadha and N. S. Satam. "A robust rapid approach to image segmentation with optimal thresholding and watershed transform," International Journal of Computer Applications, arXiv:1303.4866v1 [cs.CV], March 2013.

[10] Y. Deng and B. S. Manjunath, "Unsupervised segmentation of color-texture regions in images and video," IEEE Trans. Pattern Anal. Mach. Intell. vol. 23, no. 8, pp. 800-810, 2001.

[11] J. Dombre, N. Richard, and C. Fernandez-Maloigne, "Contentbased image retrieval and high level representation," in Proc. IST/SPIE Internet Imaging III, USA, 2002, pp.134-143.

[12] J. Dombre, N. Richard, and C. Fernandez-Maloigne, "A new representation system for content-based image retrieval: The pyramidal graph," in Proc. Color in Graphics, Image and Vision, France, 2002, pp. 384-389.

[13] L. Vincent and P. Soille, "Watersheds in digital spaces: An efficient algorithm based on immersion simulation," IEEE Trans on PAMI, vol. 13, no. 6, pp. 563-598, 1991.

[14] M. C. De Andrade, G. Bertrand, and A. A. Araujo, "Segmentation of microscopic images by flooding simulation: A catchment basins merging algorithm," in Proc. IST/SPIE Symp. on Electronic Imaging, Science \& Technology, USA, 1997, pp. 164-175.

[15] S. Philipp-Foliguet and M. B. Vieira, "Segmentation into fuzzy regions using topographic distance," presented at SIBGRAPI, Brazil 2001.

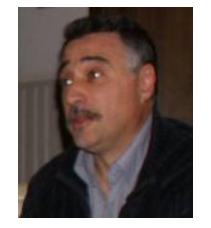

Noël Richard is assistant professor at the University of Poitiers, France, since 1993. Since 2008, he is the leader of the team of the physical, cognitive and psycho-visual axis inside the ICONES team from the XLIM Lab. His research interests include colour image analysis embedding Human Visual System model. Since 15 years, his work tends to develop new features and techniques combine them to close the image processing results from the perception or from the physical data in a metrological framework. He's the author of more than 30 international conference, journal articles and books chapter. Since 2010, his work focuses on the use of the development of new paradigm for colour morphology based on the use of distances functions in a generic purpose for non-linear processing. He coordinates several regional project, in particular for the integration of numerical tools in a total integration for regional highschool (Living-Cloud Project)

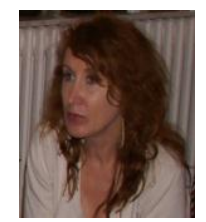

Christine Fernandez-Maloigne is Professor at the University of Poitiers, France, since 1997. She is currently co-director of XLIM laboratory, Joint Unit Research of CNRS, as well as manager of a regional research federation (MIRES), which gathers 560 researchers in region Poitou-Charentes, France, in the area of mathematics, image processing, computer graphic, computer science and communication systems. The laboratory has been selected as one the 15 Laboratories of Excellence in the area of digital Sciences in France in 2011, and it is involved in numerous national and European projects about colour imaging. Christine Fernandez-Maloigne has authored over 400 articles in peer-reviewed journals, international conferences, and books in the areas of colour imaging. She also participates to organization of numerous national and international conferences, summer schools, as well as coordination of special issues in scientific journals. Finally she is member of the National Council of Scientific Research (CoNRS), expert for the European Commission and French representative of the CIE Division 8 (International Commission of Lighting, Image Technology Division)

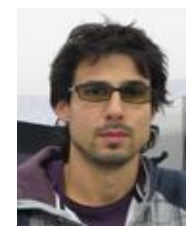

Cristian Bonanomi is research fellow at the Dept. of Computer Science of Università degli Studi di Milano. He got the Ph. D. in Computer Science in 2011. His research interest regards computer graphics, human perception, color appearance models and digital photography.

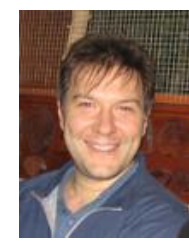

Alessandro Rizzi is Associate professor for the Dept. of Computer Science at Università degli Studi di Milano teaching Fundamentals of Digital Imaging, Multimedia Video and Human-Computer Interaction. Since 1990 he is researching in the field of digital imaging and vision. He is particularly interested in color and in the perceptual issues related with digital imaging and lighting. He is one of the founders of the Italian Color Group and member of several program committees of conferences related to color and digital imaging. He is author of more than 200 scientific papers. 